\title{
Towards visualisation of sound-scapes through dimensionality reduction
}

\author{
Aaron S.W. Wong and Stephan K. Chalup
}

\begin{abstract}
Sound-scapes are useful for understanding our surrounding environments in applications such as security, source tracking or understanding human computer interaction. Accurate position or localisation information from sound-scape samples consists of many channels of high dimensional acoustic data. In this paper we demonstrate how to obtain a visual representation of sound-scapes by applying dimensionality reduction techniques to a range of artificially generated sound-scape datasets. Linear and non-linear dimensionality techniques were compared including principle component analysis (PCA), multidimensional scaling (MDS), locally linear embedding (LLE) and isometric feature mapping (ISOMAP). Results obtained by applying the dimensionality reduction techniques led to visual representations of affine positions of the sound source on its sound-scape manifold. These displayed clearly the order relationships of angles and intensities of the generated sound-scape samples. In a simple classification task with the artificial sound data, the successful combination of dimensionality reduction and classifier methods are demonstrated.
\end{abstract}

\section{INTRODUCTION}

Sound-scapes similar to landscapes are geometric representations of an object's (a sound source's) relative position in the real world. Accurate sound-scapes can support a solid understanding of the surrounding acoustic environment. They are useful in various applications such as analysing human computer interaction, path tracking or simultaneous localisation and mapping (SLAM) in robotics and security or surveillance applications. Visualisations of sound-scapes can also serve as a cost effective alternative to vision based landscape representations, as they use microphones instead of expensive cameras.

Analogue sound can be represented by the convolution of sine waves. Digital representation of analogue sounds are obtained by sampling the analogue signal. These digital representations are high dimensional, where the number of dimensions depends on the sampling frequency and duration of the analogue signal. Many high dimensional digital representations of analogue sound recorded by different microphones at different locations at the same point in time create a sound-scape sample. The number of dimensions in a soundscape sample is dependent on the number of microphones and the number of dimensions of the digital representation.

Sound can be viewed as medium of delivering information from one point to another. This information can be sophisticated such as speech and emotion, or simple such as its primary frequency or angle of arrival or its relative position. Extracting position information from sound is natural for

Aaron S.W. Wong \& Stephan K. Chalup are with the School of Electrical Engineering and Computer Science, University of Newcastle, Australia (email: aaron.wong@studentmail.newcastle.edu.au). human beings; however, due to the high dimensionality of sound-scape samples, this is a difficult task for a computer, hence making it difficult to model a sound-scape.

Traditionally, angle of arrival estimation has been the main method of acoustic localisation. There have been many attempts through various methods to obtain the angle of arrival from sound data. These methods include psychoacoustic algorithms such as time difference of arrival (TDOA); a fast algorithm, able to obtain the angle of arrival from relative time differences between data received at sensors [1]. Another psychoacoustic technique is inter-aural level difference, where angle of arrival is obtained from relative intensity differences between the sensors [2]. These methods present correct angle of arrival estimations with the condition that the recorded signal to noise ratio (SNR) is high.

Recently statistical learning techniques have been applied to acoustic datasets, not only for localisation, but also for classification. Such techniques include neural networks, where samples of sound have been trained to track and predict the movement of a sound source to some degree of success [3]. Similarly, support vector machines have also shown some success in this field, with sound data used to create support vectors in order to classify the angle of arrival from sound [4], [5]. These methods extract localisation information from acoustic data by learning a mapping from high dimensional data to a lower dimensional representation.

Dimensionality reduction techniques aim to obtain a low dimensional representation for a given high dimensional dataset. In this paper we refer to linear dimensionality reduction techniques as methods which perform optimally for data which lies on or close to a linear manifold. Linear dimensionality reduction would perform sub-optimal if the under-laying manifold is not linear. Principle component analysis (PCA) [6] is a linear dimensionality reduction technique which preserves the covariance structure of the data, while multi-dimensional scaling (MDS) [7] is also a linear dimensionality reduction technique, but preserves the inner dot product or the pair-wise distances between the data. Isometric feature mapping (ISOMAP), a non-linear dimensionality reduction technique, is an extension of MDS (ordinal MDS [8] and classical MDS [9]), where it attempts to preserve the geodesic distances between samples. Locally linear embedding (LLE) is another non-linear dimensionality reduction technique similar to ISOMAP, but aims to preserve local structure [10]. Both non-linear dimensionality reduction techniques have shown success in benchmark tasks such as unfolding of the swiss roll and mapping of images on a submanifold. 
Recently, Errity and McKenna [11] attempted to obtain meaningful low dimensional representations of speech data for speech analysis through dimensionality reduction techniques such as PCA, MDS, ISOMAP, LLE and Lapacian eigenmaps [12]. Their experiments produced two dimensional sub-manifolds of speech data, where clusters of samples with common features such as phones and pitch can be seen from the manifold. $\mathrm{K}$ nearest neighbour ( $k$ $\mathrm{NN}$ ) classifier was then used to classify these features in the embeddings. The obtained classification results were poorer then those obtained from Mel frequency cepstral coefficient (MFCC) features, a popular method for feature extraction on acoustic data. This method did not perform up to standards for classification, but the authors' techniques produced interesting acoustic data representations such as the pitch manifold.

Errity and McKenna's work employed a single channel of sound, but in order to create a sound-scape, we require more channels. Thrun [13], presented a paper demonstrating a technique which uses multi-channel environmental sound and singular value decomposition to obtain coordinates that represent the affine position or structure of the microphones. In the present paper we will use multi-channel data but unlike Thrun, we have a different objective: to produce an affine representation of the position of a sound source (a soundscape) using dimensionality reduction techniques. We have applied two non-linear dimensionality or manifold learning techniques, ISOMAP and LLE to artificially generated acoustic data to obtain sound-scape manifolds. We compared these results with PCA and MDS.

This paper has been structured in the following manner. Section 2 will discuss the manifold learning techniques used on acoustic datasets. Dataset generation and settings are discussed in section 3. In section 4 , we will compare and contrast sound-scape manifolds obtained from techniques such as PCA, MDS, LLE and ISOMAP for dimensionality reduction on artificially generated acoustic datasets. Section 5 will generally discuss the method presented and its results. Finally, Section 6 will present the conclusions.

\section{Dimensionality Reduction Techniques}

\section{A. LLE Algorithm}

Locally linear embedding (LLE) aims to compute a low dimensional embedding of data in high dimensional space, with the property that nearby points in high dimensional space remain nearby in the low dimensional space [10]. The following is a description of the LLE algorithm:

1) Compute the neighbours of each data point $\vec{X}_{i}$. To minimize the computational complexity we select the nearest $k$ neighbours, through a distance metric such as Euclidean distance, or points within a ball of fixed radius.

2) Compute the weights $W_{i j}$ that best reconstruct each data point $\vec{X}_{i}$ from its neighbours, minimizing the cost function by constrained linear fits. The Lagrangian optimisation was applied to the constrained least squares problem, with the minimizing error function $E(W)$ for Euclidean distance being:

$$
E(W)=\sum_{i}\left|\vec{X}_{i}-\sum_{j} W_{i j} \vec{X}_{j}\right|^{2}
$$

3) Compute the vectors $Y_{i}$ that best reconstruct the weights $W_{i j}$, minimizing the embedding cost function:

$$
\Phi(Y)=\sum_{i}\left|\vec{Y}_{i}-\sum_{j} W_{i j} \vec{Y}_{j}\right|^{2}
$$

The resulting vectors $\vec{Y}_{i}$ represent global internal coordinates on a manifold.

\section{B. ISOMAP Algorithm}

ISOMAP was introduced by Tenenbaum et al. [9] and consists of three steps:

1) Once a set of data is obtained we construct a nearest neighbour graph $G$ and weight each link in $G$ by a weighting function.

2) Then we compute the complete distance matrix for all samples in $G$, using techniques such as Dijkstra's algorithm.

3) Finally, we use classical MDS to embed the data to a lower dimension.

This structure leaves a few options open, such as alternatives in the construction of the neighbourhood graph in $G$, e.g. the number of nearest neighbours, $k$ and the weighting function. For this application and simplicity we choose to use Euclidean distance as the weighting function and use $\mathrm{k}$ values ranging from 1 to 100 .

\section{DATASET Generation}

Assume we have three microphones $m_{i}, m_{j}$ and $m_{k}$ placed in an equilateral triangle configuration, with a distance between microphones denoted as $d_{m}$. A sound source is located at an angle $\theta$ degrees, at a distance $d_{s}$ away from the centre of the microphone array. Samples of the sound source signal $s$ are collected by microphones $m_{i}, m_{j}$ and $m_{k}$, to obtain recorded signals $s_{i}, s_{j}$ and $s_{k}$ respectively. These recorded signals $s_{i}, s_{j}$ and $s_{k}$ are then concatenated to create a sound-scape sample $S$. A dataset then consists of $n$ number of sound-scape samples. To create this dataset artificially, we must firstly generate the sound-scape sample $S$. This can be obtained by the concatenation of the artificially generated recorded signals $s_{i}, s_{j}$ and $s_{k}$. Generating recorded signals can be obtained by:

1) generating an original signal $s$.

2) embedding relative angular and distance information into $s$ to form the appropriate recorded signal $s_{i}$ or $s_{j}$ or $s_{k}$.

\section{A. Original Signal}

For this experiment the original sound source signal $s$ consisted of either a tone or a chirp. A tone signal is a sinusoidal wave with a constant frequency, while a chirp signal consists of a cosine wave with the wave's instantaneous frequency changing over time. Formally, $s$ can be described as: 


$$
s=a \cos (\omega(t))
$$

where $a$ is the amplitude of the original signal, $\omega(t)$ is the instantaneous frequency at time $t$. By changing $\omega(t)$, we can select between a tone or chirp. For a tone signal $\omega(t)$ is constant:

$$
\omega(t)=f t
$$

where $f$ is the frequency of the sinusoidal wave in Hertz $(\mathrm{Hz})$ at time $t$. For a chirp signal, the function $\omega(t)$ changes with time which can be described as:

$$
\omega(t)=2 \pi \mu t^{2}+2 \pi f_{0} t
$$

where $\mu$ is the rate of change of frequency, $f_{0}$ is the starting frequency of the chirp.

\section{B. Angular Information}

Angular information for sounds are obtained from the time delays between two or more recorded sound signals. To extract the angular information or the angle of arrival $\phi$, we apply time difference of arrival (TDOA) algorithms, using the sound signals, and other known information such as distance between the microphones $d$ and recorded signal properties. To assist the dataset generation process we apply a reverse TDOA to obtain the number samples $n$ between two recorded signals at the desired sound source arrival angle of $\theta$ degrees:

$$
n=f s \times c \times d \cos \left(90^{\circ}-\theta\right)
$$

where $f s$ is the sampling frequency of the recorded signal and $c$ is the speed of sound. We then embed the angular information denoted by the sample shift $n$ into the original signal $s$ by shifting an identical $s$ by $n$ samples. This forms two of the three recorded signals needed for the sound-scape sample.

The third signal was created by selecting one of the already generated signals as a reference. We repeat the process to calculate the time delay to embed into the third signal. This was achieved by using the original angle of arrival $\theta$ combined with angle between the vector formed from the new microphones and the $x$-axis. Once three recorded signals $s_{i}, s_{j}$ and $s_{k}$ were obtained, we concatenate these to form a sound-scape sample $S$.

\section{Distance Information}

Relative distance can be represented by the intensity difference between microphones. We assume that microphones $m_{i}$ and $m_{j}$ were a distance $d_{i j}$ apart and recorded an acoustic source emitted sound $s$, as $s_{i}$ and $s_{j}$. The received signals would differ from each other not only by a time delay but also by attenuation. The relationship between attenuation, distance between the source and microphone $d_{s \mu}$ can be described as:

$$
s_{\mu} \propto \frac{1}{\left(d_{s \mu}\right)^{2}}
$$

where $\mu=i, j, k$ is the relative recorded signal. The recorded sound signal $s_{\mu}$ is inversely proportional to the distance between the source and microphone squared. By calculating the distance between each source and microphone $d_{s i}$ and $d_{s j}$, we then scale the received signal proportionally to the calculated distance. Hence microphones $m_{i}$ and $m_{j}$ with a distance $d_{i j}$ apart, will have different intensities if the $d_{s i}$ and $d_{s j}$ are not equal.

Relative distance information can be embedded into the dataset by scaling the amplitude $a$ of an original signal in Eq. 3. Each sound-scape sample is inversely scaled to represent the relative distance.

\section{EXPERIMENTS \& RESULTS}

\section{A. Sound-scape Angular Manifold}

Using the above dataset generation technique, we constructed a dataset which represented sound-scape samples obtained from an array of microphones. This dataset simulated an acoustic source moving at a constant distance $d$ away from the microphones, with angles ranging from $0^{\circ}$ to $360^{\circ}$ at an interval of $0.5^{\circ}$. The recorded path represents a sound source in circular motion around the microphones. PCA, MDS, LLE and ISOMAP were used to obtain low dimensional representations of this dataset, with nearest neighbour parameters $k$ ranging from 1 to 100 trialled for both LLE and ISOMAP.

The obtained results with the highest quality are shown in Fig. 1. PCA obtained results that showed clusters of data which did not have a meaningful representation. MDS revealed a sparse low dimensional representation which also had poor relevance to localisation. The best visual representation obtained from LLE was using the nearest neighbour parameter $k=2$, which displayed a path of angles in sequential order in a two dimensional embedding. ISOMAP obtained the best low dimensionality visual representation, showing a close representation of the path, a circle.

ISOMAP with parameter $k=2$ led to a reduced dimensional representation of the sound-scape dataset. This enables us to clearly visualise the path of the sound source on a two dimensional plane. This method results in affine positions of the sound sources or simulated samples of acoustic data recorded from microphones. Although ISOMAP produced as expected the shape of a circle, several small loops can be recognised along the circle in Fig. 1. At this stage we have no explanation for these loops other than that they maybe caused by to ISOMAP's high sensitivity to noise.

\section{B. Sound-scape Intensity Manifold}

Intensity information was incorporated into dataset firstly by selecting three different distances between microphones and the sound source. Then using Eq. 7, we calculated and scaled the intensity of the time shifted signal for each simulated channel. This generated three different sound sources moving in a circular motion each positioned at a particular distance away from the microphones. Similarly to the previous experiment, dimensionality reduction techniques 


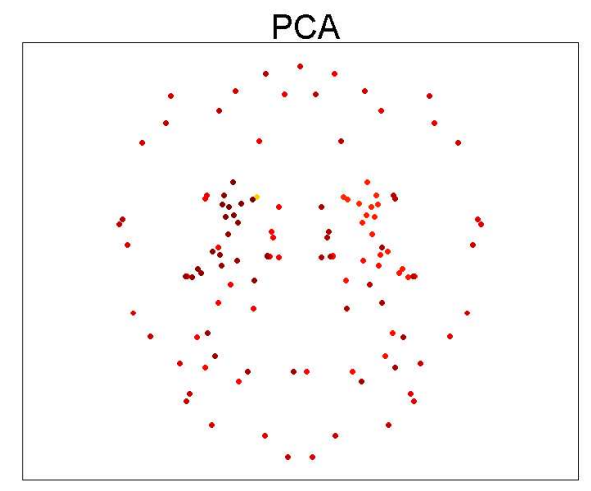

LLE

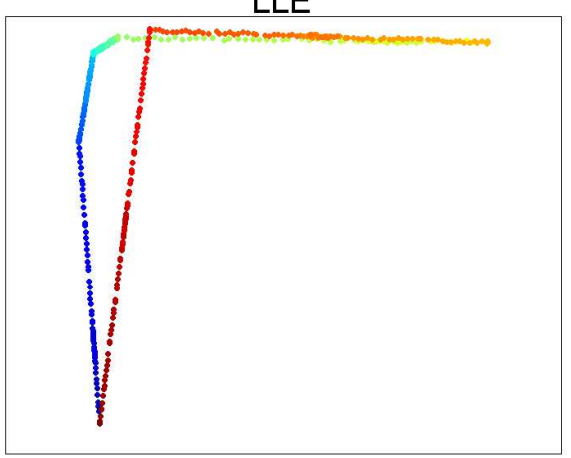

Non-Metric MDS

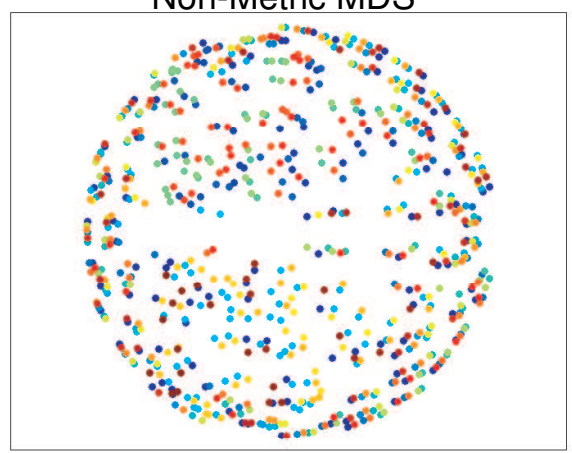

ISOMAP

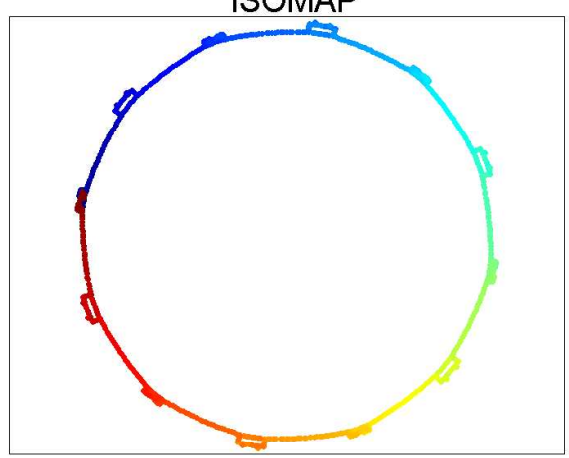

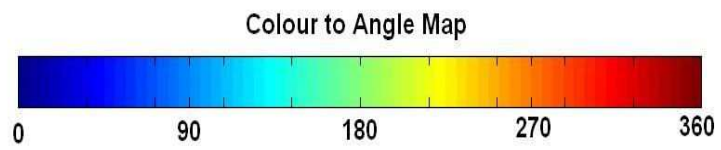

Fig. 1. Sound-scape Angular Manifolds: Results obtained from dimensionality reduction techniques on an acoustic sound-scape dataset generated to represent a source in circular motion around the microphones. Top: Shows the results for linear dimensionality reduction consisting of: PCA and non-metric MDS. Bottom Left: Displays best result obtained from LLE. Bottom Right: Resulting manifold obtained with ISOMAP has the highest resemblance to the manifold which underlies the simulated dataset.

were applied to the dataset. Trials for both LLE and ISOMAP used varying parameter $k$ ranging from 1 to 100 .

Results obtained are displayed in Fig. 2. PCA produced a poor two dimensional representation. The LLE representation showed some relation to intensity; as the intensity of the sound-scape sample increased, the further the sample would move away from the centre of the manifold. MDS produced a two dimensional representation that can visually separate the intensities or distance relationship of the dataset with ease, however the representation did not show an acceptable angular representation. ISOMAP obtained a manifold with the greatest meaning; as samples increased their intensity, the sample would be positioned relatively further away from the centre, while keeping its angular relationship. This created three prominent bands of sound-scape positions, each representing intensity at different levels.

\section{Spiral Motion}

Acoustically, spiral motion can be described as a sound source moving in a circular motion away (or towards) the microphone array at the centre. Sound-scape samples of this nature can be similarly simulated with the algorithm in previous section. We incorporated an increasing linear distance change between the sound source and the microphones. As the sound source moves further away from the microphones the received signal's intensity inversely decreases by the square of the distance between the sound source and the microphones. Using this property, we generated a sound-scape dataset that simulated a sound source spiralling away from the microphones. We then applied dimensionality reduction techniques similarly to previous experiments to obtain sound scape representations. 

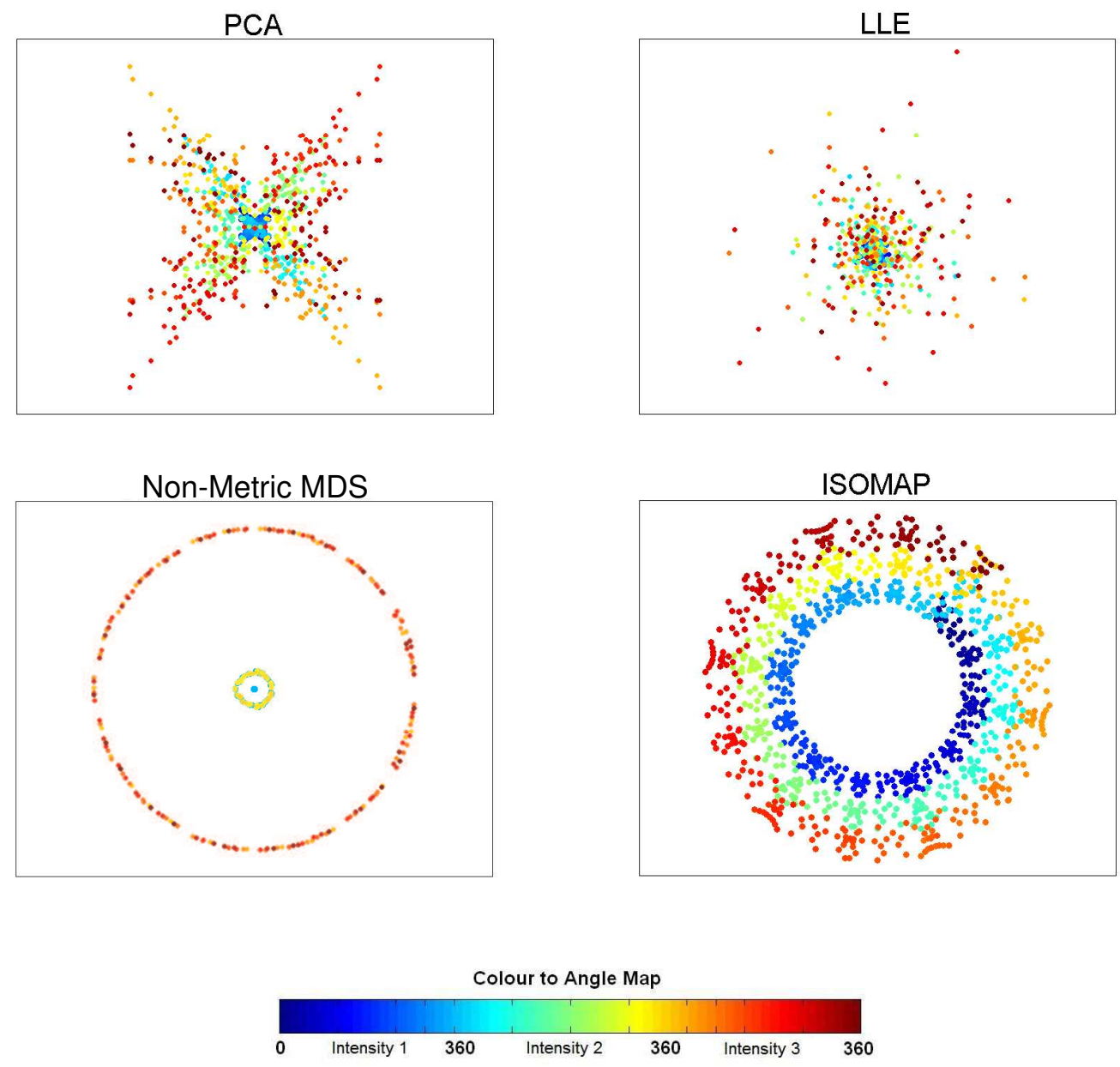

Fig. 2. Sound-scape Intensity Manifolds: Results obtained from dimensionality reduction techniques on an acoustic sound-scape dataset generated to represent a sound source in circular motion around the microphones at different distances. Top: Shows the results for PCA and LLE. Bottom displays results obtained from MDS and ISOMAP.

MDS and ISOMAP with parameter $k=2$ were applied to the data set Fig. 3. Both resultant figures display the position of the sound-scape samples relative to each another. The manifold obtained by ISOMAP showed a relevant representation of the sound-scapes dataset, a sound source moving in a spiral-like path. Results obtained by MDS showed a more visually appealing spiral path when compared with ISOMAP. The MDS manifold displayed a clearer sound-scape sample intensity relationship. However, ISOMAP revealed a linearly increasing representation of the sample distance away from the centre of the manifold and is proportional to the distance $d_{s \mu}$. This showed a closer representation of the simulated sound source's path with the additional benefit of better distinguishing the inner points when compared with MDS.

\section{Multiple Sources}

Two sound sources with different signals (a chirp and a tone) were generated for this dataset. Each sound source was then modified to represent sound in spiral motion, moving away from the microphones, as the previous section described. Once the sound sources were generated, they were combined to create a multi-source sound-scape dataset. Once this multi-source sound-scape dataset was obtained, we then applied dimensionality reduction techniques PCA, MDS and ISOMAP to obtain manifolds that represent the data, similarly to our previous experiments.

First, PCA and non-metric MDS were applied to the data. The resulting manifolds are displayed in Fig. 4. These figures show an interesting representation of the sound-scape dataset. The two dimensional PCA representation of the soundscape dataset shows two distinct clusters of data points. The 

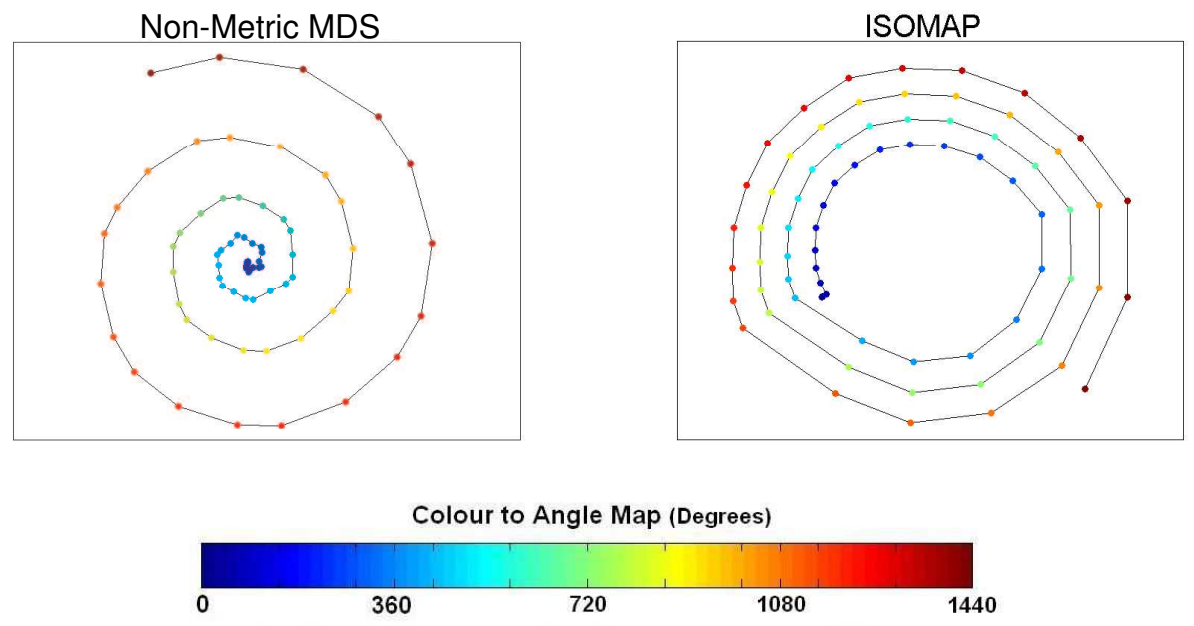

Fig. 3. Sound-scapes manifolds of spiral motion: MDS and ISOMAP were applied to obtain the above results.
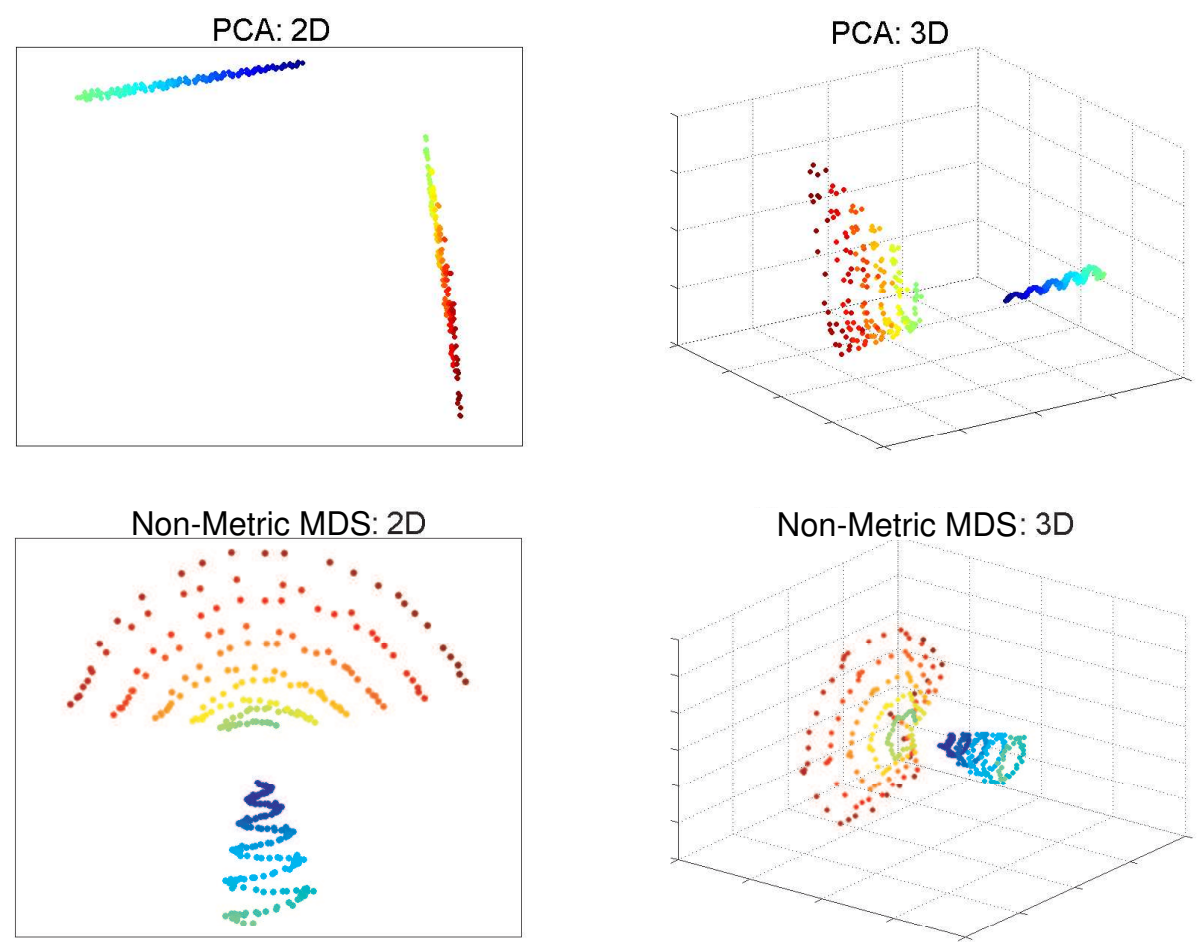

Non-Metric MDS: 3D
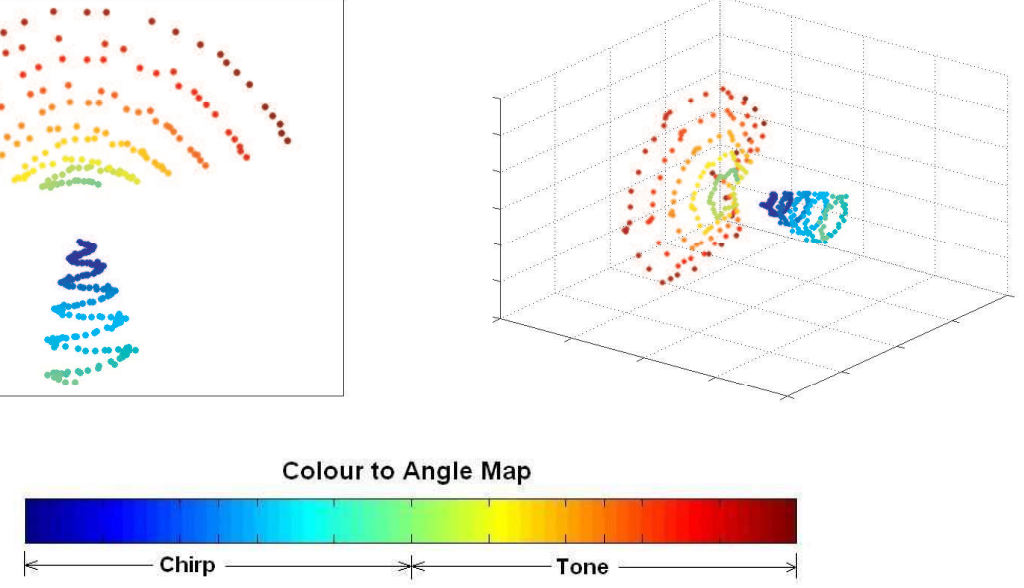

Fig. 4. Multiple Source Sound-scape Manifolds: Results obtained from traditional dimensionality reduction techniques: Top: PCA in two dimensional representation and PCA in in three dimensional representation. Bottom: MDS in two dimensional representation and MDS in in three dimensional representation are shown above. 
equivalent parameters in MDS showed two clusters of data in an alternating path. The corresponding representations of the results in three dimensions show two spirals, with MDS displaying the results clearer then PCA.

Next, ISOMAP was applied to the generated multiple source sound-scape dataset, it returned a reduced dimensional manifold representing the dataset (see Fig. 5). With a small nearest neighbour parameter $k=8$, we obtained a manifold that represented the relative angular position of each sample, while a large nearest neighbour parameter $k=50$ allowed us to visually separate the datasets into two different spirals, similar as with PCA and MDS.

The obtained results of ISOMAP with the higher nearest neighbour $k=50$ (see Fig. 5. middle) displayed two distinct clusters of samples when viewed in two dimensions. However, when viewed in three dimensions, it revealed the a clear representation of each cluster in a spiral motion (see Fig. 5. bottom). These resulting manifolds show appropriate sound-scape representations.

\section{E. Application: Classification}

Sound source classification is an important topic for the field of acoustics. One of the attributes that can assist classification is through its location with respect to the listener or microphones. This can be achieved efficiently in special cases through an extension of the prior experiments. We illustrate this using a straightforward example; assuming that sufficient information is known about the source, a specific sound-scape sample was taken from a specific class. We used this information to label its corresponding low dimensional co-ordinates.

We applied a $k$ nearest neighbour $(k-\mathrm{NN})$ classifier and support vector machines (SVM) [14] to obtain classification for the rest of the dataset. Generally, the $k$-NN algorithm selects the majority of labelled sound-scape samples closest to the samples in question using a measure of Euclidean distance. This simulates the ability to classify sound sources using additional directional or position information provided through visual inspection by the user.

The results obtained from applying both $k$-NN classifier and SVM to a random set of low dimensional data points obtained from ISOMAP are shown in Fig. 6. Results for $k$ NN have a classification rate of $99.2 \%$ and $100 \%$ for SVM in this application.

\section{DISCUSSION}

This paper has verified the possibility of obtaining soundscape representations through dimensionality reduction using artificially generated sound-scape data. A potentially useful application of this method in classification is demonstrated using a simple example. However, there are limitations that need to be addressed before the proposed approach could be employed, for example in real world applications.

The proposed method currently generates a manifold using the dimensionality reduction techniques with a dataset at one time. However, it would be more useful in applications such as security, to have this algorithm running online updating a
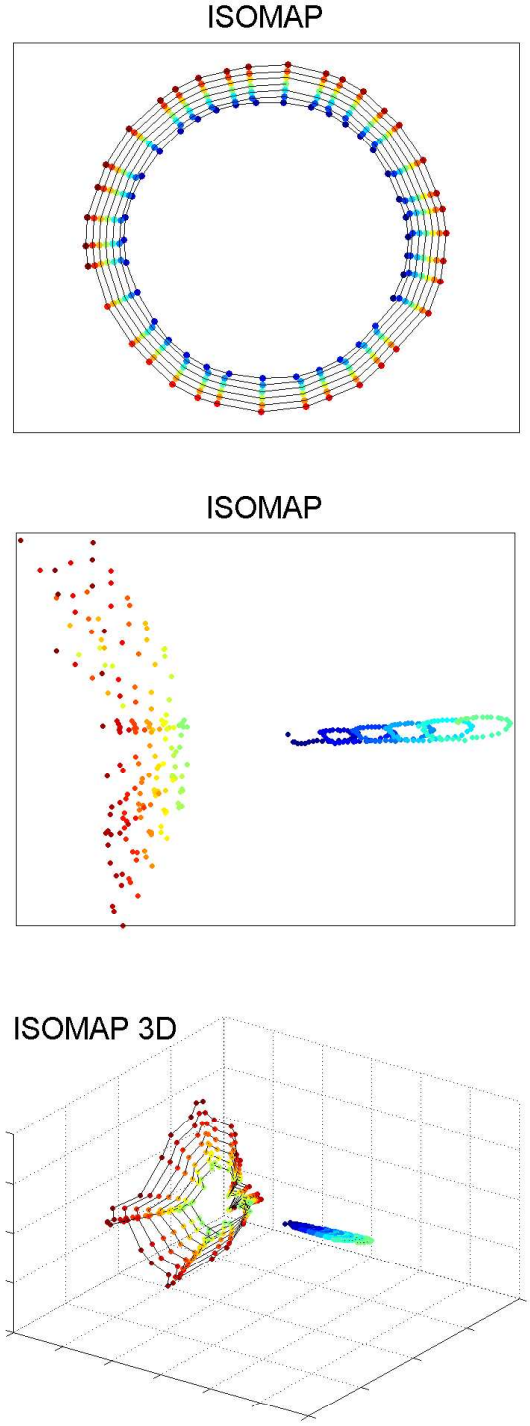

Colour to Angle Map

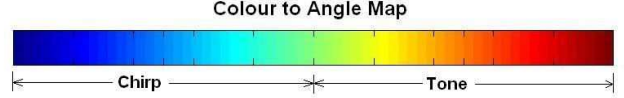

Fig. 5. Multiple source sound-scape manifolds: The same multiple source sound-scape dataset as in Fig. 4. was used, but ISOMAP was applied to obtain the above results. Top: Representation obtained using a lower $k$ parameter of 8 . Middle and Bottom: Results obtained from using higher $k$ parameter of 50 in two and three dimensional representation are respectively shown above. 

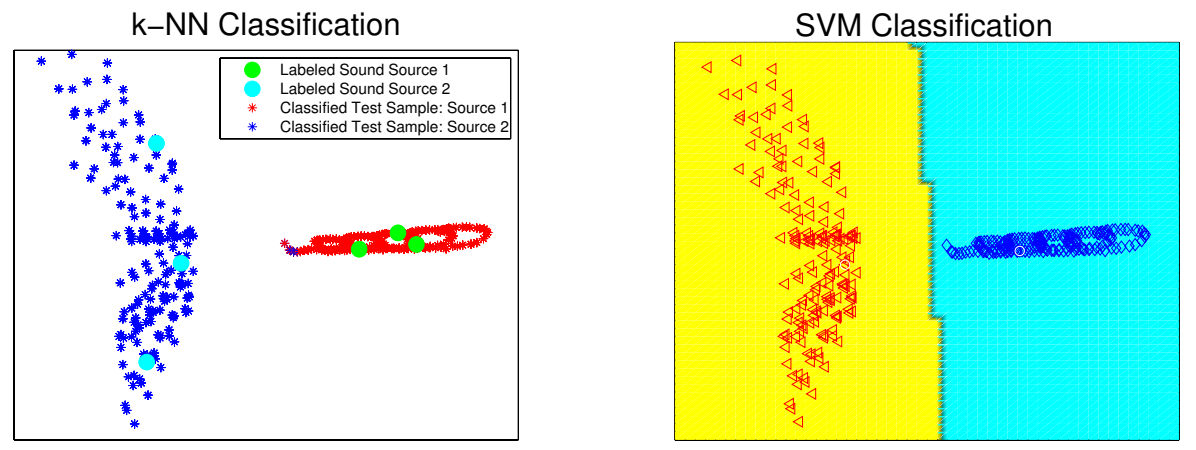

Fig. 6. Example of classification combined with dimensionality reduction: Assuming six samples are labelled, we are able to classify the rest of the data set, Left: using $k$-NN with an accuracy of $99.2 \%$, and Right: using SVM obtaining an accuracy of $100 \%$.

manifold by processing a sound-scape sample at one time. Problems such as these are addressed by [15].

The current framework is not scalable to the size of the sound-scape dataset. Large numbers of sound-scape samples may take a long time to run with dimensionality reduction techniques such as ISOMAP. These problems can be levitated by using recent algorithms such as landmark ISOMAP [16].

In real world, acoustic datasets will always contain noise. This artificial dataset is generated to have minimum noise, but the affects of noise are shown in the resulting manifold (Fig. 1). To assist in alleviating this problem, noise reduction or noise cancellation algorithms could be applied as a preprocessing stage to sound-scape dataset. This may help further minimise noise before dimensionality reduction techniques are applied to obtain a better sound-scape manifold.

\section{CONCLUSIONS}

This paper has presented a method of obtaining soundscape visualisations though dimensionality reduction. We have compared the quality of the results obtained by various dimensionality reduction techniques such as PCA, MDS, LLE and ISOMAP when applied to the task of obtaining sound-scape manifolds from several artificially generated sound-scape datasets. In several cases it was possible to extract sound-scape manifolds, that displayed appropriate angular and intensity relationships. ISOMAP produced acceptable representations of the path of a sound source in spiral motion. In the case of multiple sources, we obtained an angular representation for lower values of nearest neighbour parameter $k$. However for higher values of $k$ we obtained representations which clustered similar data points together. In combination with standard classification methods, this allowed us to achieve high classification rates on sound source classification example task. Future extensions to this study may address noisy data and real world applications.

\section{REFERENCES}

[1] Y. Toyoda, J. Huang, S. Ding, and Y. Liu, "Environmental sound recognition by multilayered neural networks," in Computer and Information
Technology, 2004. CIT '04. The Fourth International Conference on. Dept. of Inf. Syst., Aizu Univ., Japan, Sept 2004, pp. $123-127$.

[2] R. Birchfield, S.T. Gangishetty, "Acoustic localization by interaural level difference," in Acoustics, Speech, and Signal Processing, Proceedings. (ICASSP '05). IEEE International Conference on, vol. 4. Dept. of Electr. \& Comput. Eng., Clemson Univ., SC, USA, 2005.

[3] H. Murray, J. Wermter, and S. Erwin, "Auditory robotic tracking of sound sources using hybrid cross-correlation and recurrent networks," in Intelligent Robots and Systems, 2005. (IROS 2005). 2005 IEEE/RSJ International Conference on, 2005, pp. 3554- 3559.

[4] Y. Y. Chen and S. H. Lai, "Audio-visual information fusion for svmbased biometric verification," in Cellular Neural Networks and Their Applications, 2005 9th International Workshop on. Dept. of Comput. Sci., Nat. Tsing Hua Univ., Hsinchu, Taiwan, May 2005, pp. $300-$ 303.

[5] C. Lei, S. Gunduz, and M. Ozsu, "Mixed type audio classification with support vector machine," in Multimedia and Expo, 2006 IEEE International Conference on. Department of Computer Science, Hong Kong University of Sci. and Tech., July 2006, pp. $781-784$.

[6] I. T. Jolliffe, Principal Component Analysis. New York: SpringerVerlag, 1986.

[7] T. F. Cox and M. A. A. Cox, Multidimensional Scaling. Chapman \& Hall/CRC, 2001.

[8] J. B. Tenenbaum, "Mapping a manifold of perceptual observations," in Advances in Neural Information Processing Systems 10. Denver, Colorado, United States, 1998, pp. 682 - 688.

[9] J. B. Tenenbaum, V. D. Silva, and J. C. Langford, "A global geometric framework for nonlinear dimensionality reduction," Science, vol. 290 , no. 5500, pp. 2319-2323, 2000.

[10] L. K. Saul and S. T. Roweis, "Think globally, fit locally: Unsupervised learning of low dimensional manifolds," Journal of Machine Learning Research 4, vol. 4, pp. 119-155, 2003.

[11] A. Errity and J. McKenna, "An investigation of manifold learning for speech analysis," in Spoken Language Processing (Interspeech 2006 ICSLP). Antwerp, Belgium: Pittsburgh PA, USA, August 2006.

[12] M. Belkin and P. Niyogi, "Laplacian eigenmaps for dimensionality reduction and data representation," in Neural Computation, vol. 15, 2003, pp. 1373-1396.

[13] S. Thrun, "Affine structure from sound," in Advances in Neural Information Processing Systems 18, Y. Weiss, B. Schölkopf, and J. Platt, Eds. Cambridge, MA: MIT Press, 2005, pp. 1353-1360.

[14] J. Ma, Y. Zhao, and S. Ahalt, "Osu svm classifier matlab toolbox (version 3.00)," Software, 2003, matlab interface to OSU SVM ToolBox.

[15] Y. Bengio, J.-F. Paiement, and P. Vincent, "Out-of-sample extensions for lle, isomap, mds, eigenmaps, and spectral clustering," Universite de Montreal, Montreal, Quebec, Canada, Tech. Rep. 1238, 2003.

[16] V. de Silva and J. B. Tenenbaum, "Global versus local methods in nonlinear dimensionality reduction," in Advances in Neural Information Processing Systems 15., 2002, pp. 721 - 728. 